\title{
Chordomas; Crush Intraoperative Analysis
}

\author{
Tena-Suck $\mathrm{ML}^{1^{*}}$, Estrada-Natoli $\mathrm{L}^{2,3}$, Corona-Cobian $\mathrm{LE}^{4}$ and Torral- Rizo $\mathbf{V H}^{3}$ \\ ${ }^{1}$ Department of Neuropathology, National Institute of Neurology and Neurosurgery, México City, Mexoico \\ ${ }^{2}$ Service of Cytopathology, (CITOCAP), Hospital Ángeles del Pedregal, México City, México \\ ${ }^{3}$ Department of Pathology, Orthin Laboratory, Mexico City, Mexico \\ ${ }^{4}$ Department of Pathology, General Hospital of the west, Zapopan Jalisco México
}

"Corresponding author: Tena Suck ML, Department of Neuropathology, National Institute of Neurology and Neurosurgery "Manuel Velasco Suárez", Av Insurgentes Sur 3877 Col. La Joya, Delegación Tlalpan CP 14269, Mexico City, Mexico, Tel: (525) 56063822; Fax: (525) 54240808; E-mail: mltenasuck@ gmail.com.mx

Rec date: Feb 24, 2015; Acc date: May 16, 2015; Pub date: May 18, 2015

Copyright: (c) 2015 Tena-Suck ML, et al. This is an open-access article distributed under the terms of the Creative Commons Attribution License, which permits unrestricted use, distribution, and reproduction in any medium, provided the original author and source are credited.

\begin{abstract}
Chordomas are known by primary malign tumors that have formed from primitive notochord remains and exhibit different epithelial properties. Morphologically these cells have distinct borders; the nucleus displays a monotony and blandness. It was found few cases of pleomorphism, atypia, anaplasia and hipercromatic nuclei. Vacuoles were seen in the cytoplasm. This study was realized during January 1995 until June 2005, with 22 surgeries of chordomas and intraoperative crush. The background was dirty with myxoid appearance, myxoid matrix growing in sheets or cord and had vacuoles showed more evidence of physaliferous cells. Chondroid chordoma showed vacuolated cells as well as other cells with eosinophilic cytoplasm and myxoid background. Single cells were observed only in two cases and high cellularity was observed in $8(42 \%)$ cases. Two cases were frank errors, only one had a partial correlation. The percentage of mistakes was $30 \%$ and our diagnosis accuracy was $70 \%$. The correlation with clinical details and radiological findings were helpful in improving the accuracy rate. There was no differentiation between cytomorphological features smear of intracranial tumours and the sacrococcygeal location. Intraoperative analysis of chordomas could be helpful to have a good diagnosis and to have a better surgical resection.
\end{abstract}

Keywords: Chordomas; Crush intraoperative study; Smears; Brain tumours; Cytomorphology

\section{Introduction}

Chordomas are a type of tumors which originate from the remnants of the notochord. Originally chordomas represent $1-4 \%$ of all primary malignant bone tumors [1]. It is constituted $5 \%$ of all neoplasms and $86 \%$ of bone tumors [2]. About one-half of chordomas are located in the sacrococcygeal region and approximately $30 \%-35 \%$ are present at the base of the skull [1]. However, the chordomas can occur anywhere along the vertebral column. Chordomas are rarely observed in children or adolescents. Men are affected more frequently than women [1]. Originally chordoma it is considered as an intermediate grade malignant bone tumour [3], as low tendency to metastasis and has a poor prognosis in long-term follow-up. Metastases are seen in 5-40\% of chordoma cases $[2,3]$.

There are three histological variants of chordoma: classical (or conventional) chondroid and dedifferentiated [1,2]. The chordoma cells are constituted have small round nuclei and abundant vacuolated cytoplasm, sometimes described as physaliferous (having bubbles or vacuoles) $[1,2]$.

Macroscopically, these tumors are lobulated gelatinous and brownish-grey in colour, and occasionally appear translucent [1-4]. Histologically, these tumors are characterized by the presence of physaliphorious cells, which are very rich in mucin and glycogen [1-3]. Tissue section of the aspirate sample shows sheets of vacuolated "physaliphorious" cells, classic of chordoma [2-4].
The Intraoperative smear cytology provides a rapid and reliable intraoperative diagnosis besides to guidance the neurosurgeon during surgical resection and lesion targeting. It also helps the surgeon to monitor and modify the approach at surgery. The smear cytology is of great value in intraoperative consultation of central nervous system tumours [5].

The smear technique is challenging for neuropathologists where rapid and accurate diagnosis is to be given on small biopsies and conducted to assess the usefulness, accuracy and the diagnostic pitfalls of smear diagnosis [5]. Squash smear technique is a very reliable and rapid method of intraoperative diagnosis. Knowledge of clinical and neuroimaging details helps the experienced neuropathologists to improve the diagnostic accuracy [5].

An intraoperative method ideal should be accurate, rapid and should allow preservation of tissue for paraffin-embedded sections. The frozen section technique is less popular for brain biopsies as it uses a substantial amount of tissue and produces freezing artefacts $[5,6]$.

We retrospectively reviewed 22 cases from patients diagnosed by crush intraoperative smear of chordoma focusing on the cytomorphological features of the tumors. Cytologicaland histological correlation.

\section{Material and Methods}

\section{Clinical cases}

It was performed a search with the database from Department of Neuropathology at National Institute of Neurology and Neurosurgery 
Page 2 of 5

(period 1995 until 2005) in the Mexico City. In this study were included 22 cases of chordomas. All the tumours intraoperatory were analyzed. Samples were obtained by direct and visible masses or under computed tomography (CT) or under imaging guidance in the deepseated brain neoplasms. Cytological studies included evaluation of aspirated material from the primary tumor and the correlation ship with the definitive biopsy diagnosed.

A histologic and cytological correlative retrospective review was performed to assess the ability of cytology to render an accurate and specific diagnosis of this malignancy. All samples were obtained by direct and visible masses or under computed tomographic (CT) or under imaging guidance in the deep-seated brain neoplasms (Figure 1a).
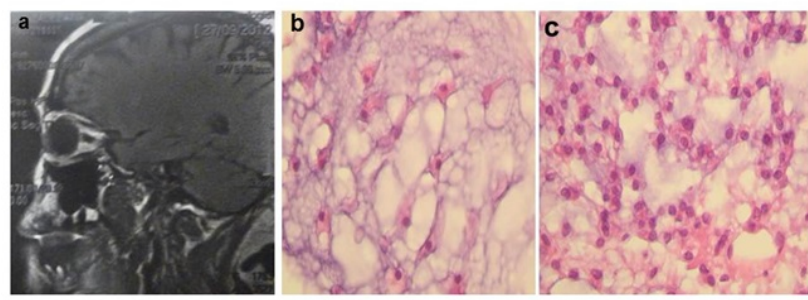

Figure 1: (a) Showed an MRI in sagittal imaging that showed an enhancement tumour in skull base and in (b) Histologically, these tumours are characterized by the presence of physaliphorious cells, which are very rich in mucin and glycogen, in (c) observed a chordoid chordoma features (H\&E 400X).

For all specimens, slides were air dried immediately for hematoxylin and eosin stain. Smear preparation, and preliminary microscopic interpretation were performed immediately in all cases. A cell block preparation was obtained for most of the cases that were embedded in paraffin. There was histological confirmation in all cases.

In the cases were evaluated for the following cytomorphological parameters: cellularity (scanty, moderate, high, cellular arrangement or in single cells), sheets or cluster formation, prominent nucleoli (present or absent), nuclear molding (present or absent), nuclear-tocytoplasmic ratio (low, moderate, high), and background material (inflammatory, necrotic, bloody, mucinous, or clean). Discrepancies in interpretation were resolved by forum of discussion. Overall, interobserver agreement was high. Cytological features and causes for the discrepancy of the final diagnosis were reviewed and noted. At the time of review, all cases had tissue correlation with the surgical resection specimen of the primary tumor.

\section{Results}

Twenty-two cases were included in this study, with clinical and radiological diagnosis of chordomas. This cases were underwent biopsy and intraoperative study crushed.

The specific tumor subtype on cytology, in cases in which an attempt was made to further refine the diagnosis, was erroneous in four cases. The only false-negative diagnoses were in 3 cases and resulted in definite diagnosis on insufficient material. The cytological findings are summarized in Table 1.
Nineteen cases corresponded to classic type and there was chordoid type. Sixteen cases $(73 \%)$ were male and $6(27 \%)$ female, aged from 15 to 86 years (median 49 year). Tumor localization: 12 (55\%) cases were located intracranial and 10 (45\%) in sacrococcygeal location.

\begin{tabular}{|c|c|c|}
\hline & $\begin{array}{l}\text { Classic } \\
\text { Chordoma } \\
n=19(\%)\end{array}$ & $\begin{array}{l}\text { Chondroid } \\
\text { Chordoma } \\
n=3(\%)\end{array}$ \\
\hline \multicolumn{3}{|l|}{ Cellularity } \\
\hline Scanty & $1(59)$ & $1(33)$ \\
\hline Moderate & $10(53)$ & $1(33)$ \\
\hline High & $8(42)$ & $1(33)$ \\
\hline \multicolumn{3}{|l|}{ Arrangement } \\
\hline Single cells & $1(5)$ & 0 \\
\hline Sheets & $8(42)$ & $1(33)$ \\
\hline Clusters & $10(53)$ & $2(67)$ \\
\hline \multicolumn{3}{|c|}{ Cellular prominent nucleoli } \\
\hline Present & $8(42)$ & $2(67)$ \\
\hline Absent & $11(58)$ & $1(33)$ \\
\hline \multicolumn{3}{|l|}{ Nuclear molding } \\
\hline Present & $12(63)$ & $1(33)$ \\
\hline Absent & $7(37)$ & $2(67)$ \\
\hline Atypia & $8(42)$ & $3(100)$ \\
\hline Pleomorphism & $5(26)$ & $3(100)$ \\
\hline \multicolumn{3}{|c|}{ Background material } \\
\hline Fibrillary & $1(5)$ & $1(33)$ \\
\hline Granular & $19(100)$ & $3(100)$ \\
\hline Haemolytic & $3(16)$ & $1(33)$ \\
\hline Myxoid & $19(100)$ & $3(300)$ \\
\hline Bloody & $3(16)$ & $3(100)$ \\
\hline Inflammation & $5(26)$ & $1(33)$ \\
\hline
\end{tabular}

Table 1: Cytomorphological characteristic of chordoma smears.

The neoplastic cells are very fragile; these cells usually disintegrate and break at the time of preparation (Figures $2 \mathrm{a}$ and $2 \mathrm{~b}$ ). The cells exhibit epithelial this properties, have distinct cell borders (Figure $2 b$ ), abundant cytoplasm and are adherent to each other (Figure 2c). This can be viewed in isolated cell (Figure 2d) or in sheets or in slightly eosinophilic cord (Figure 2d).

The nucleus displays a monotony and blandness (Figure 2e). Pleomorphism, atypia, anaplasia and hyperchromatic nuclei were minimal. Vacuoles were seen in the cytoplasm (Figure 2e). The background found was usually dirty with myxoid appearance, myxoid matrix growing in sheets or cord and had vacuoles (Figure 2f). Chondroid chordoma always showed myxoid background (Figures 3a 
and 3b), some cells showed eosinophilic vacuoles (Figure 3c), as well as other cells with eosinophilic cytoplasm (Figure 3d) and perinuclear halos are seen in some cells (Figure 3d). The cytoplasm found was usually thin, fine granulated and radiated (Figure 3e), also was found nuclear atypia with anisonucleosis (Figure 3f). The generalities of smear were observed in Table 1. Single cells were observed just in two cases (9\%), high cellularity was observed in eight cases (42\%) and moderated cellularity was in twelve cases (54.5\%). The error percentage was $30 \%$.

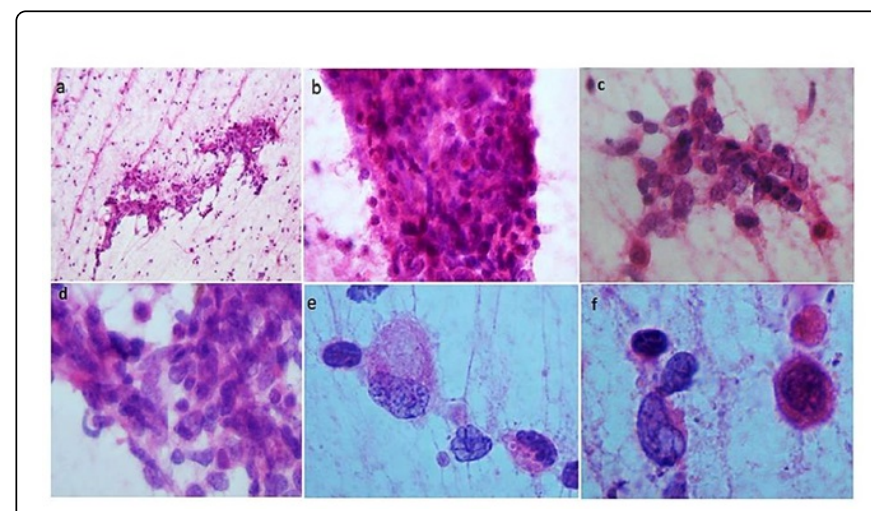

Figure 2: (a) Chordoma smears at low power observed a few cells in a myxoid background (H\&E 200X). (b) Moderate cellularity observed with predefined of mixed background (H\&E 200X). (c) Singles cells demonstrates the seemingly syncytial appearance of this tumour cells (H\&E 400X). And in (d), observed a high cellularity, cell with an epithelial appearance with abundant clear cytoplasm. The smear produces thick cytoplasmic bridges among a small cell group (H\&E 400X). (e) Smear showed single cells with abundant clear and vacuolated cytoplasm and homogeneous nuclei (H\&E 400X). (f) Close up observed two cell types of benign appearance (H\&E 1000X).

Correlation with clinical details and radiological findings were helpful in improving the accuracy rate. The results were an accuracy of diagnosis of correlation from $70 \%$. Overall, interobserver agreement was considered high. There was no differentiation between cytomorphologic smear of intracranial tumours vs sacrococcygeal location, neither classical vs chondroid subtype. However, chondroid type showed more eosinophilic cells than classic type.

\section{Discussion}

Eisenhardt and Cushing were the first to describe the histological diagnosis in brain tumours in 1930 of touch preparations stained with supravital dyes for the rapid diagnosis of a brain tumour at the time of the surgery [7]. The method was provided reliable in the past with an accuracy rate of $95 \%$ [7]. This large retrospective analysis of smears in neurosurgical practice highlights the usefulness of this technique. It is a simple, reproducible and reliable technique which gives good cytological detail for making reasonably accurate diagnosis of lesions of the CNS. Various authors used different stains like haematoxylin and eosin and May-Grunwald-Giemsa, and Papanicolaou stains [7-10] etc. The reported diagnostic accuracy of cytological smears ranged from $75 \%$ to $94 \%$ in various series [7-10]. To conclude, the squash smear technique is a very accurate and rapid method of intraoperative diagnosis, but adequate clinical history, neuroimaging details and the intraoperative impression of the neurosurgeon if provided, helps the neuropathologists to improve the diagnostic accuracy $[7,8]$. Our reported diagnostic accuracy in chordomas was about $70 \%$. Clinical history, location and imaging can help in looking for specific features.

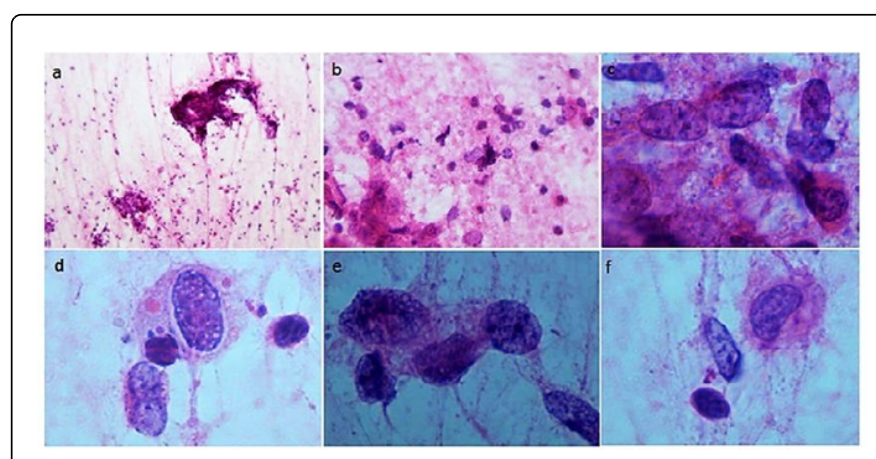

Figure 3: Chondroid Chordoma at low power smears showed tumour cell that was arranged in sheets, clusters with chondroid stroma. (b) Neoplastic cells that are very fragile and disintegrate background and break at the time of preparation. The background is dirty with myxoid appearance, myxoid matrix (H\&E 200X). (c) The cell exhibit epithelial proprieties have distinct cell borders, abundant cytoplasm and are adherent to each other with granular appearance, in (d) observed isolated cells with eosinophilic cytoplasm and clear appearance of halos perinuclear and the vascular cytoplasm (H\&Ex400X). (e) Observed some isolated cells that cytoplasm is usually thin, fine granulated and radiated, and in (f) also nuclear atypism with anisonucleosis and seen (H\&E 1000X).

The smear technique is a rapid diagnostic method and its interpretation is based on a small sample of tissue. Inadequate clinical and imaging data can contribute to wrong diagnosis. The diagnostic accuracy was highest in tumors. However, partial correlation was due to grades and mixed tumors. The tumors which required more tissue, special stains and/or immunohistochemical confirmation for final diagnosis posed problems for diagnosis on smear [5,6]. It is believed that our results are supportive of the accuracy of the procedure and comparable to other reported series

Clinical prerequisites for a cytological diagnosis of brain tumors included; age of the patients, tumor location, and tumor size were considered. The indications for a fine needle aspirations included midline lesions that are inaccessible to direct surgical remove or tend to infiltrate adjacent vital structures [7-9].

Crushed smears as well needle biopsy have been reported a pitfall in diagnosis included assessment of non-specific gliosis, necrosis and notochord cells in this case. Cytology of smears and crush preparation also offer some advantages to being frozen sections; better preservation of cellular detail and minimizes the adverse changes as necrosis. The disadvantages are the loss of tumour architectural detail in crush smears.

The classic chordoma consisted of multiple lobules that were separated by thin fibrous septa and that showed cords or strands of atypical physaliferous cells set within an abundant myxoid matrix [9]. Metachromatic stroma in between large physaliferous cells containing bubbly, vacuolated cytoplasm and small round nucleus. In contrast, the benign lesions consisted of intraosseous sheets of bland physaliferous cells without any extracellular matrix [6-9]. 
Page 4 of 5

The cytological features of chondroid chordoma observed in intraoperative crush and touch cytology revealed round or stellate cells distributed in a mucoid background without a typical epithelial cordlike arrangement [7-10]. The cytological features of classical chordoma include conspicuous extracellular matrix in the background. Polygonal cells dissociated and arranged in small groups, were identified in all cases [10]. Stellate and cuboidal cells often contained intracytoplasmic vacuoles of varying sizes and round or oval nuclei and showed slight cellular pleomorphism [10]. Intranuclear inclusions, mitotic Figureures, and anisonucleosis were prominent features of some cases published [10]. Physaliferous cells were also prominently found in these cases. In addition, the case with anaplastic features showed very bizarre cells with profound multinucleation and the presence of intranuclear cytoplasmic inclusions [6,7]. May Giemsa stain has been demonstrated that the mucoid matrix and vacuolated cytoplasm of the tumour cells $[8,9]$, additionally, crush preparations were effective in demonstrating well-differentiated chondroid elements $[8,9]$. In our results, some cases presented some discrepancies between cytological features and definitive histological diagnosis, in some case immunohistochemistry could be a fully help.

Immunohistochemistry demonstrated cytoplasmic staining for low vs high-molecular-weight cytokeratins, vimentin, and epithelial membrane antigen, while glial fibrillary acidic protein and carcinoembryonic antigen have been reported as negative [10] Immunocytochemistry with positivity for S-100 protein and cytokeratins, have been an essential adjunct in the cytological diagnosis of chordoma and helped in distinguishing it from other chondroid tumours [7-9].

We must considered differential diagnosis, especially tumours with clear or vacuolated cells (clear cell meningioma, clear cell ependymoma, haemangioblastoma liposarcoma, lipoma, metastatic adenocarcinoma, and renal carcinoma), tumours with myxoid stroma (metastatic adenocarcinoma, myxoid liposarcoma, chondrosarcoma) and tumour with chondroid differentiation (chondrosarcoma, parachordoma, enchondroma). Thus, in cerebral localization based primarily affecting cranial as well as in the sacrococcygeal region. The microscopic hallmark of these tumours is the presence of characteristic large cells with numerous cytoplasmic vacuoles known as physaliferous (Greek: droplet bearing) cells [7-9]. Tumours of the clivus and metastasis to the clivus are very rare. Metastasis of the neoplasm may occur in $10-40 \%$ of the cases. Because of its unusual frequency, the diagnosis of chordoma may be difficult to render, especially on fine-needle aspiration biopsy. This distinction in the case of metastases can be made easily, where correlation of previous histology has been done and/or ancillary studies have been performed $[10,11]$.

The presence of classic physaliferous cells on fine needle aspiration is an essential for the diagnosis of chordoma, vacuolated cells have been missed diagnosed as metastatic lesions or adenocarcinomas [10-15]. Cellular chordomas can appear epithelioid in the sacrum and they may resemble metastatic squamous or transitional cell carcinomas. This distinction in the case of metastases can be made easily, where correlation of previous histology has been done and/or ancillary studies have been performed.

Appropriate immunocytochemical studies with clinical and cytological evaluation is recommended for avoiding misinterpretation with adenocarcinoma. Nuclear immunoreactivity of chordomas for S-100 protein appears to be a significant immunomarker for the differential diagnosis, although some adenocarcinomas may also be immunoreactive for S-100 protein [8-15].

Hemangioblastoma is a rare benign vascular tumour commonly seen in the cerebellum. There is a striking histologic similarity between a cellular variant of hemangioblastoma and metastatic renal cell carcinoma [16], both tumours showed clear and vacuolated cells and the diagnosis can be missed due to these morphological similarities [16].

Liposarcoma (LS) smears are composed in different proportions of round, spindle and clear cells, lipoblasts, and myxoid and vascular arborizing structures [17]. Pure well-differentiated LS were frequently composed of lipoblasts, and round or spindle cells were occasionally seen [17]. Dedifferentiated and sclerosing liposarcoma is composed of spindle or round cells, but lipoblasts are also occasionally present [17]. Well-differentiated LS should be distinguished from hibernoma and spindle cell lipoma, and myxoid LS from myxoma, myxoid chondrosarcoma, chordoma, myxoid leiomyosarcoma, and myxoid malignant fibrous histiocytomas [7-10].

The presence of myxoid material in neoplasms should be included extra skeletal myxoid chondrosarcoma, chordoma, myxoid adenocarcinoma, myxoma, lipomatous tumours, nerve sheath tumours, smooth muscle tumours, gastrointestinal stromal tumour and other sarcomas [6-10].

Recognition of the cytological features characteristic may allow the distinction to be made on fine needle aspiration biopsy. Because of the prominent mucinous elements and papillary fronds, mixopapillary ependymoma may mimic other myxoid or papillary tumours cytological [5-7,18-20]. These help to distinguish it from other chondrogenic tumours and metastatic mucous-producing carcinoma [6-9]. The cytological criteria for differentiating chordoma from chondrosarcoma, mucinous metastatic adenocarcinoma and mixopapillary ependymoma include the vacuolated cells and the presence of bland nuclear features [21]. Exfoliative cytology of the sputum showed cohesive, epithelioid clusters composed of palestained, broad cytoplasm with a lacelike pattern, minimal nuclear atypia with anisonucleosis and characteristic mucoid substances [7-10].

Chondroid meningioma is a rare variant of meningioma that bears a striking histological resemblance to chordoma and has greater likelihood of recurrence [20].

The cytomorphological features of rhabdomyosarcoma (RMS) include small round to ovoid cells, and occasionally pleomorphic cells, with myxoid stroma [20]. However, in most cases studied; there are no specific cytological features [20-22].

The diagnosis of chordoma may be difficult to render, especially on fine-needle aspiration biopsy (FNAB) and crush smears. The cytological features of classical chordoma include conspicuous extracellular matrix in the background. Polygonal cells, dissociated and in small groups, were identified in all cases. Physalipherous cells were also prominently found in these cases. In addition, the case with anaplastic features showed very bizarre cells with profound multinucleation and the presence of intranuclear cytoplasmic inclusions in chondroid type.

Conclusion; However, a clear-cut distinction of chordoma from other neoplasms is of utmost importance, since the prognosis and treatment of the patient will depend on the final diagnosis. In our cases, there were no differences between cranial vs sacrococcygeal 
Citation: Tena-Suck ML, Estrada-Natoli L, Corona-Cobian LE, Torral-Rizo VH (2015) Chordomas; Crush Intraoperative Analysis. J Cytol Histol 6: 328. doi:10.4172/2157-7099.1000328

Page 5 of 5

location, however, chordoid chordoma showed atypia in Physalipherous cells than classic type. Maybe can say that classic chordoma showed vacuolar cells and chondroid type showed atypical and eosinophilic Physalipherous cells.

\section{References}

1. Heffelfinger MJ, Dahlin DC, MacCarty CS, Beabout JW (1973) Chordomas and cartilaginous tumors at the skull base. Cancer 32 410-420.

2. Mirra JM, Della Rocca C, Nelson SD, Mertens F (2002) In Pathology and genetics of tumours of soft tissue and bone.

3. Naka T, Boltze C, Kuester D, Samii A, Herold C, et al. (2005) Intralesional fibrous septum in chordoma: a clinicopathologic and immunohistochemical study of 122 lesions. Am J Clin Pathol 124: 288-294.

4. Zimnoch L, Kozielec Z, Lewko J, Baltaziak M, Mariak Z (1997) Intracranial chordomas; histochemical and immunohistochemical examinations. Neurol Neurochir Pol 31: 89-101.

5. Bleggi-Torres LF, De Noronha L, Schneider Gugelmin E, et al. (2001) Accuracy of the smear technique in the cytological diagnosis of 650 lesions of the Central Nervous System. Diagn Cytopathol 4: 293-295.

6. Roessler K, Dietrich W, Kitz K (2002) High diagnostic accuracy of cytologic smears of central nervous system tumors. A 15-year experience based on 4172 patients. Acta Cytol 46: 667-674.

7. Eisenhardt L, Cushing H (1930) Diagnosis of intracranial tumors by intravital technique. Am J Pathol 6: 541-552.

8. Walaas L, Kindblom LG (1991) Fine-needle aspiration biopsy in the preoperative diagnosis of chordoma: a study of 17 cases with application of electron microscopic, histochemical, and immunocytochemical examination. Hum Pathol 22: 22-28.

9. Firlik KS, Martinez J, Lunsford LD (1999) Use of cytological preparations for the intraoperative diagnosis of stereotactically obtained brain biopsies: a 19-year experience and survey of neuropathologists. J Neurosurg 91: 454-458.

10. Silverman JF, Silverman JF, Timmons RL, et al. (1986) Cytologic results of fine needle aspiration biopsies of Central Nervous System. Cancer 58 1117-1121.
11. Finley JL, Silverman JF, Dabbs DJ, West RL, Dickens A, et al. (1986) Chordoma: diagnosis by fine-needle aspiration biopsy with histologic, immunocytochemical, and ultrastructural confirmation. Diagn Cytopathol 2: 330-337.

12. Nijhawan VS, Rajwanshi A, Das A, Jayaram N, Gupta SK (1989) Fineneedle aspiration cytology of sacrococcygeal chordoma. Diagn Cytopathol 5: 404-407.

13. Gupta RK, AlAnsari AG (1996) Value of image-guided needle aspiration cytology in the assessment of thoracolumbar and sacrococcygeal masses. Acta Cytol 40: 215-221.

14. Kfoury H, Haleem A, Burgess A (2000) Fine-needle aspiration biopsy of metastatic chordoma: case report and review of the literature. Diagn Cytopathol 22: 104-106.

15. Hall WA, Clark HB (1995) Sacrococcygeal chordoma metastatic to the brain with review of the literature. J Neurooncol 25: 155-159.

16. Ingold B, Wild PJ, Nocito A, Amin MB, Storz M, et al. (2008) Renal cell carcinoma marker reliably discriminates central nervous system haemangioblastoma from brain metastases of renal cell carcinoma. Histopathology 52: 674-681.

17. Klijanienko J, Caillaud JM, Lagace R (2004) Fine-needle aspiration in liposarcoma: cytohistologic correlative study including welldifferentiated, myxoid, and pleomorphic variants. Diagn Cytopathol 30: 307-312.

18. Ng WK, Khoo US, Ip P, Collins RJ (1998) Fine needle aspiration cytology of myxopapillary ependymoma. A case report. Acta Cytol 42:1022-1026

19. Kumar ND, Misra K (1990) Fine needle aspiration cytodiagnosis of subcutaneous sacrococcygeal myxopapillary ependymoma. A case report. Acta Cytol 34: 851-854.

20. Salinero E, Beltran L, Costa JR (2004) Intraoperative cytologic diagnosis of chordoid meningioma. A case report. Acta Cytol 48: 259-263.

21. Taraszewska A, Bogucki J, Andrychowski J, Koszewski W, Czernicki Z (2003) Clinicopathological and ultrastructural study in two cases of chordoid glioma. Folia Neuropathol 41: 175-182.

22. Agarwal S, Agarwal T, Agarwal R, Agarwal PK, Jain UK (2000) Fine needle aspiration of bone tumors. Cancer Detect Prev 24: 602-609. 